\title{
MicroRNA-146a downregulates the production of hyaluronic acid and collagen I in Graves' ophthalmopathy orbital fibroblasts
}

\author{
WEI LIU, CHAO MA, HAO-YU LI, LAN CHEN, SHAN-SI YUAN and KAI-JUN LI \\ Department of Ophthalmology, The First Affiliated Hospital of Guangxi Medical University, \\ Nanning, Guangxi 530021, P.R. China
}

Received November 17, 2019; Accepted June 11, 2020

DOI: $10.3892 / \mathrm{etm} .2020 .9165$

\begin{abstract}
The present study aimed to investigate the effect of microRNA (miR)-146a on the secretion of hyaluronic acid (HA) and collagen I in Graves' ophthalmopathy (GO) orbital fibroblasts, and identify potential novel targets for the clinical treatment of GO. Orbital fibroblasts were extracted from orbital connective tissue, and primary cells were identified via immunohistochemistry. The levels of HA and collagen I in orbital fibroblasts of non-GO controls and patients with $\mathrm{GO}$ were examined via reverse transcription-quantitative PCR (RT-qPCR). miR-146a was overexpressed or inhibited in primary orbital fibroblasts via lentiviral infection, and the levels of HA and collagen I following miR-146a overexpression or inhibition were detected via ELISA and RT-qPCR. The results indicated that the mRNA expression of HA and collagen I was higher in orbital fibroblasts from patients with GO compared with the non-GO cohort. Overexpression of miR-146a reduced, and inhibition of miR-146a increased the production of HA and collagen I in GO orbital fibroblasts. In conclusion, it was demonstrated that miR-146a downregulated the secretion of HA and collagen I in GO orbital fibroblasts in vitro, which may affect glycosaminoglycan aggregation and collagen deposition in GO.
\end{abstract}

\section{Introduction}

Graves' Ophthalmopathy (GO), which is also known as thyroid eye disease, is an autoimmune disease that is caused by thyroid dysfunction and seriously affects the quality of life of patients suffering from it (1). The principal clinical manifestations of GO include ocular protrusion, eyelid contraction, exposure keratitis, restricted eye movement and oppressive optic neuropathy (2). The pathogenesis of GO has not yet been fully

Correspondence to: Professor Kai-Jun Li, Department of Ophthalmology, The First Affiliated Hospital of Guangxi Medical University, 6 Shuangyong Road, Nanning, Guangxi 530021, P.R. China E-mail: 463105616@qq.com

Key words: Graves' ophthalmopathy, hyaluronic acid, collagen I, microRNA, pathogenesis elucidated, is complex and has been indicated to involve both genetic and environmental factors (3). It has been reported that $\mathrm{T}$ cell-induced inflammation, glycosaminoglycan (GAG) aggregation and fibrosis originating from orbital fibroblasts are the primary pathological mechanisms of GO (4). Histological evidence has revealed that GO was consistently accompanied by the infiltration of lymphocytes in the posterior globules, the accumulation of GAG [primarily hyaluronic-acid (HA)] and collagen deposition, which resulted in the development of connective tissue hyperplasia and fibrosis $(5,6)$. These pathological alterations have been reported to be associated with orbital fibroblasts (7).

In the pathogenesis of GO, orbital fibroblasts have been reported to produce large amounts of HA and collagen I (8). HA is the principal component of GAG and remains anchored to the cell surface after its synthesis via binding to either hyaluronan synthase or other surface receptors, while an amount of HA is also cleaved by hyaluronidase and is released in the extracellular matrix (9). Owing to the potent hydrophilic nature of HA, its accumulation has been indicated to accelerate the expansion of orbital tissues (10). Collagen I is considered to be a marker of fibrosis (11). Fibrocytes, which express CD34 and $\mathrm{C}-\mathrm{X}-\mathrm{C}$ chemokine receptor type 4 , have been reported to produce collagen I and infiltrate tissues in response to multiple chemokines, including C-X-C motif chemokine 12 (12), which may result in fibrosis in orbital tissues.

MicroRNAs (miRNAs/miRs) are non-coding, single-stranded RNA molecules that are 22 nucleotides in length, which have been indicated to regulate gene expression at the post-transcriptional level (13). miRNAs have been reported to serve an important role in the pathogenesis of numerous diseases, including cancer and infectious diseases (14), and it has been suggested that they may serve as novel targets for disease treatment (15). In recent years, several studies have described the role of miRNAs in inflammatory autoimmune diseases (16-18). miR-146a has been reported to serve a role in a variety of autoimmune diseases, including rheumatoid arthritis, systemic lupus erythematosus and osteoarthritis (19). Previous studies have indicated that miR-146a regulated the expression of inflammatory factors in GO orbital fibroblasts $(19,20)$. The current study aimed to investigate the effect of miR-146a on the production of HA and collagen I in GO orbital fibroblasts, and indicated that miR-146a may be used as a novel target for the treatment of GO. 


\section{Materials and methods}

Reagents. HA (cat. no. m1026554) and collagen I (cat. no. m1029092) ELISA kits were purchased from Shanghai Enzyme-linked Biotechnology Co., Ltd. Antibodies against vimentin (cat. no. D220268), S100B (cat. no. D161409), myoglobin (cat. no. D222721), keratin 17 (cat. no. D220232) and desmin (cat. no. D162991) were purchased from Shanghai Shenggong, Biology Engineering Technology Service, Ltd. A Histostain-SP kit (cat. no. SP-0023) was purchased from BIOSS. TRIzol ${ }^{\circledR}$ reagent was supplied by Invitrogen, Thermo Fisher Scientific, Inc. (cat. no. 15596026), Inc. miR-146a mimics, miR-146a inhibitor, control mimics, control inhibitor and Polybrene Infection Reagent were purchased from Shanghai GeneChem Co., Ltd. Primers for miR-146a and U6 were purchased from Takara Biotechnology Co., Ltd. The primer sequence for miR-146a was withheld from the supplier (cat. no. MAQ472). Primers for HA and collagen I, GAPDH were purchased from Shanghai Shenggong Biology Engineering Technology Service, Ltd.

Patients. Patients were recruited according to the Bartley diagnostic criteria and clinical activity scores (CAS) (21), where $\geq 4$ points indicated active GO lesions and $<4$ points indicate that GO is inactive. The orbital connective tissue of patients treated at the First Affiliated Hospital of Guangxi Medical University from January 2018 to October 2019 was obtained for the current study (Table I). A total of 6 patients with inactive GO (CAS <4) with adipose tissue after orbital decompression and 4 patients (non-GO patients) with eyeball removal or upper lid blepharoplasty were included in the current study. Patients who had previously used immunosuppressive agents and exhibited other autoimmune diseases (including asthma, chronic inflammation and HIV), a recent history of trauma or an active infection within six months prior to surgery were excluded. Written informed consent was obtained from all participants prior to inclusion. The current study was approved by the Ethics Review Committee of the First Affiliated Hospital of Guangxi Medical University.

Cell culture and infection. Orbital fat or connective tissues from patients with GO and non-GO patients were collected under aseptic conditions during surgery (eyeball removal or upper lid blepharoplasty), placed immediately into a $50 \mathrm{ml}$ centrifuge tube containing DMEM high glucose culture fluid (Gibco; Thermo Fisher Scientific, Inc.) and 1\% penicillin/streptomycin on ice. The tissue specimens were washed three times with PBS under sterile conditions. The blood vessels, which were visible on the tissue blocks, were removed using sterile ophthalmic micro scissors. Following three washes with PBS, the tissue was cut into pieces of $\sim 0.5 \mathrm{~mm}^{3}$ and transferred to a $15 \mathrm{ml}$ centrifuge tube. Pancreatin digestion solution containing EDTA (Dalian Meilun Biology Technology Co., Ltd.) was added and the centrifuge tube was incubated at $37^{\circ} \mathrm{C}$ $\mathrm{m}$ for $15 \mathrm{~min}$. Following the addition of DMEM containing 10\% FBS (Shanghai Shuangru Biotechnology Co., Ltd.) to terminate the digestion, the tube was centrifuged at $25^{\circ} \mathrm{C}$ for $15 \mathrm{~min}$ at $1,200 \mathrm{xg}$. The suspension was subsequently filtered using a 200 Mesh stainless steel filter (Beijing Solarbio
Science \& Technology Co., Ltd.) to remove incompletely digested tissue pieces, and was centrifuged at $1,200 \mathrm{x} \mathrm{g}$ for $15 \mathrm{~min}$ at room temperature. The cell pellet was dissolved in DMEM containing 20\% FBS and 1\% penicillin/streptomycin, seeded in cell culture flasks at a density of $40,000 \mathrm{cells} / \mathrm{cm}^{2}$ and placed in an incubator at $37^{\circ} \mathrm{C}$ with $5 \% \mathrm{CO}_{2}$. After 2-3 days, the cell culture medium was replaced with fresh DMEM containing $10 \% \mathrm{FBS}$ and $1 \%$ penicillin/streptomycin. Immunohistochemical identification was performed to determine whether the extracted cells were orbital fibroblasts. The expression of HA and collagen I in orbital fibroblasts of patients with GO and non-GO patients was detected via reverse transcription-quantitative PCR (RT-qPCR). The fibroblasts were seeded in a 6-well plate at a concentration of $5 \times 10^{4}$ cells $/ \mathrm{ml}$ and infected with a lentivirus carrying miR-146a mimics, miR-146a inhibitor, control mimics or control inhibitor (each $20 \mathrm{nmol} / \mathrm{l}$ ) for $12 \mathrm{~h}$. Then, the cell culture medium was replaced with fresh DMEM containing $10 \% \mathrm{FBS}$ and $1 \%$ penicillin/streptomycin. After $72 \mathrm{~h}$, a green fluorescence signal could be observed under a fluorescence microscope, which indicates successful infection. Uninfected orbital fibroblasts were removed using complete medium (DMEM containing 10\% FBS and 1\% penicillin/streptomycin) containing puromycin $(2 \mu \mathrm{g} / \mathrm{ml})$ for 3-4 days. Then, replace the cell culture medium with fresh DMEM containing 10\% FBS and $1 \%$ penicillin/streptomycin and continue culturing for 2-3 days. At this time, fibroblasts can be used for subsequent experimentation. The morphology of the orbital fibroblasts and the infection efficiency were observed using a light microscope (magnification, x100) and an inverted fluorescence phase contrast microscope (magnification, x100), respectively. The expression of miR-146a in the orbital fibroblasts of each group was detected via RT-qPCR, and the expression of HA and collagen I was detected via RT-qPCR and ELISA. After 2-3 days, subsequent experimentation was performed.

Immunohistochemistry (IHC). IHC was employed to examine the expression of vimentin, desmin, myoglobin, keratin 17 and S100B. A total of $3-5 \times 10^{4} / \mathrm{ml}$ primary orbital fibroblasts were seeded on slides. After the cells were attached, the supernatant was discarded, the cells were washed three times with PBS and subsequently incubated with $4 \%$ paraformaldehyde (Solarbio, China) at $4^{\circ} \mathrm{C}$ overnight. The cell slides were washed $3 \mathrm{x}$ with PBS and incubated with $3 \% \mathrm{H}_{2} \mathrm{O}_{2}$ in deionized water for $10 \mathrm{~min}$ at room temperature. Following three washes with PBS, the slides were blocked with whole goat serum included in the IHC kit (cat. no. IHC001; Beijing Biosynthesis Biotechnology Co., Ltd.) was used at $37^{\circ} \mathrm{C}$ for $10-20 \mathrm{~min}$. The blocking solution was removed, and the primary antibodies (vimentin, desmin, myoglobin, keratin 17 and S100B; each, 1:400) were added and incubated at $4^{\circ} \mathrm{C}$ overnight. After washing three times with PBS, the biotin-labeled goat anti-rabbit IgG (included in the IHC kit) was added and incubated at $37^{\circ} \mathrm{C}$ for $30-60 \mathrm{~min}$. The cell slides were washed three times with PBS, and 3,3'-diaminobenzidine reagent was added for color development. After 4-10 min of hematoxylin counterstaining at room temperature, the excess dye solution was removed, and the slides were washed with water for $5 \mathrm{~min}$, dehydrated and sealed with a neutral gum. A light microscope (magnification, x200) was used to observe slides. 
Table I. Clinical information of the patients included in the current study.

\begin{tabular}{|c|c|c|c|c|c|c|}
\hline & $\begin{array}{c}\text { Age } \\
\text { (years) }\end{array}$ & Sex & Smoker & $\begin{array}{l}\text { Duration of } \\
\text { GO (years) }\end{array}$ & CAS & $\begin{array}{l}\text { Surgical } \\
\text { treatment }\end{array}$ \\
\hline \multicolumn{7}{|c|}{ A, Patients with GO } \\
\hline 1 & 61 & Female & $\mathrm{N}$ & 0.5 & $0 / 7$ & Decompression \\
\hline 2 & 48 & Female & $\mathrm{N}$ & 0.5 & $1 / 7$ & Decompression \\
\hline 3 & 52 & Female & $\mathrm{N}$ & 1 & $1 / 7$ & Decompression \\
\hline 4 & 49 & Male & $\mathrm{Y}$ & 1 & $3 / 7$ & Decompression \\
\hline 5 & 57 & Male & $\mathrm{N}$ & 20 & $1 / 7$ & Decompression \\
\hline 6 & 63 & Male & $\mathrm{N}$ & 0.6 & $1 / 7$ & Decompression \\
\hline
\end{tabular}

B, Non-GO control subjects

\begin{tabular}{lclcccc}
\hline & $\begin{array}{c}\text { Age } \\
\text { (years) }\end{array}$ & Sex & Smoker & $\begin{array}{c}\text { Duration of } \\
\text { GO (years) }\end{array}$ & CAS & $\begin{array}{c}\text { Surgical } \\
\text { treatment }\end{array}$ \\
\hline 1 & 35 & Female & $\mathrm{N}$ & $\mathrm{n} / \mathrm{a}$ & $\mathrm{n} / \mathrm{a}$ & Eye evisceration \\
2 & 21 & Male & $\mathrm{N}$ & $\mathrm{n} / \mathrm{a}$ & $\mathrm{n} / \mathrm{a}$ & Eye evisceration \\
3 & 50 & Male & $\mathrm{Y}$ & $\mathrm{n} / \mathrm{a}$ & $\mathrm{n} / \mathrm{a}$ & Eye evisceration \\
4 & 33 & Female & $\mathrm{N}$ & $\mathrm{n} / \mathrm{a}$ & n/a & Upper lid blepharoplasty \\
\hline
\end{tabular}

CAS, clinical activity score; GO, Graves' orbitopathy; n/a, not applicable; N: no; Y, yes.

Table II. Primer sequences used in reverse transcription-quantitative PCR.

\begin{tabular}{lll}
\hline Gene name & \multicolumn{1}{c}{ Forward primer } & \multicolumn{1}{c}{ Reverse primer } \\
\hline HA & 5'-CACGTAACGCAATTGGTCTTGTCC-3' & 5'-CCAGTGCTCTGAAGGCTGTGTAC-3' \\
COL1A2 & 5'-CTGGACCTCCAGGTGTAAGC-3' & 5'-TGGCTGAGTCTCAAGTCACG-3' \\
GAPDH & 5'-GACATGCCGCCTGGAGAAAC-3' & 5'-AGCCCAGGATGCCCTTTAGT-3' \\
U6 & 5'-GGAACGATACAGAGAAGATTAGC-3' & 5'-TGGAACGCTTCACGAATTTGCG-3'
\end{tabular}

HA, hyaluronic acid; COL1A2, collagen Ia2.

$R T-q P C R$. Orbital fibroblast total RNA from the miR-146a mimics, miR-146a inhibitor, control mimics, control inhibitor groups was extracted with TRIzol ${ }^{\circledR}$ reagent. The concentration and quality of RNA were determined using an ultra-differential photometer (NanoDrop ${ }^{\mathrm{TM}}$ 2000; Thermo Fisher Scientific, Inc.). The RNA samples were reverse transcribed using PrimeScript RT reagent kit with gDNA Eraser from Takara Biotechnology Co., Ltd. $\left(37^{\circ} \mathrm{C}\right.$ for $60 \mathrm{~min} ; 85^{\circ} \mathrm{C}$ for $5 \mathrm{~min}$ ) and qPCR was performed using SYBR Premix Ex Taq II from Takara Biotechnology Co., Ltd. (one cycle of $95^{\circ} \mathrm{C}$ for $10 \mathrm{sec}, 95^{\circ} \mathrm{C}$ for $5 \mathrm{sec}$ and $60^{\circ} \mathrm{C}$ for $20 \mathrm{sec}$; and 40 cycles of $95^{\circ} \mathrm{C}$ for $60 \mathrm{sec}, 55^{\circ} \mathrm{C}$ for $30 \mathrm{sec}$ and $95^{\circ} \mathrm{C}$ for $30 \mathrm{sec}$ ). The relative gene expression was normalized using real-time quantitative $\mathrm{PCR}$ and the $2^{-\Delta \Delta \mathrm{Cq}}$ method (22). The expression of mir-146a was normalized to that of U6. The primer sequences for HA and collagen I are presented in Table II, the expression of which was normalized to GAPDH expression.
ELISA. Following infection, the culture supernatant of the miR-146a mimics, miR-146a inhibitor, control mimics, control inhibitor group was collected and centrifuged at $4{ }^{\circ} \mathrm{C}$, for $10 \mathrm{~min}$ at $800 \mathrm{x}$ g. The expression levels of collagen I and HA in the culture supernatant of confluent orbital fibroblasts were detected in triplicates using commercially available Human HA ELISA kits and Human collagen I ELISA kits according to the manufacturer's protocol.

Statistical analysis. All experiments were repeated three times and data were expressed as the mean \pm standard deviation. Statistical analysis was performed using SPSS v22.0 (IBM Corp.). Statistical differences between two groups were analyzed using the independent Student's t-test, while multiple group comparisons were made using one-way ANOVA followed by Fisher's least significant difference method for pairwise comparisons. $\mathrm{P}<0.05$ was considered to indicate a statistically significant difference. 


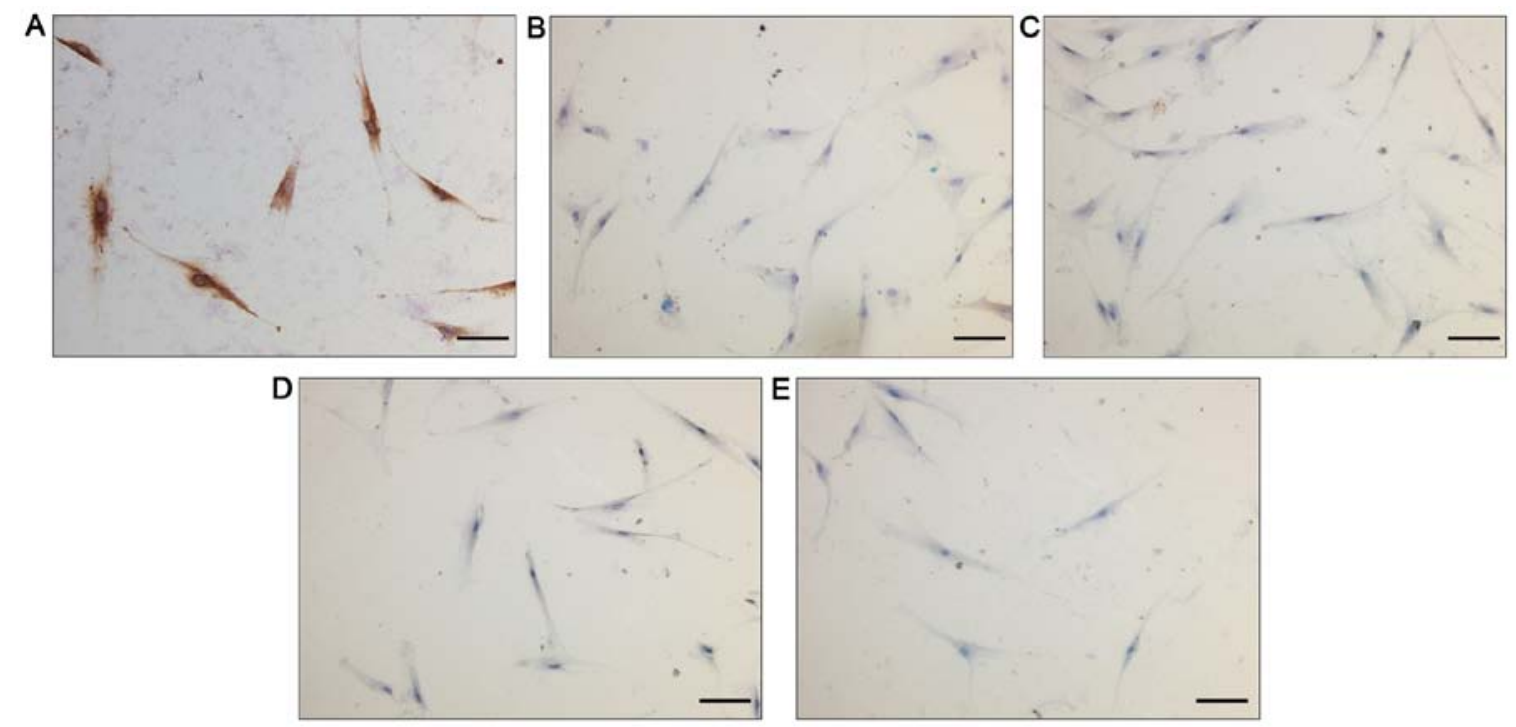

Figure 1. Immunohistochemical examination of primary orbital fibroblasts. The staining of (A) vimentin, (B) desmin, (C) myoglobin, (D) keratin 17 and (E) S100B was examined. Brown color indicates a positive cytoplasmic staining, while blue color indicates a negative staining. Scale bars, $50 \mu \mathrm{m}$.
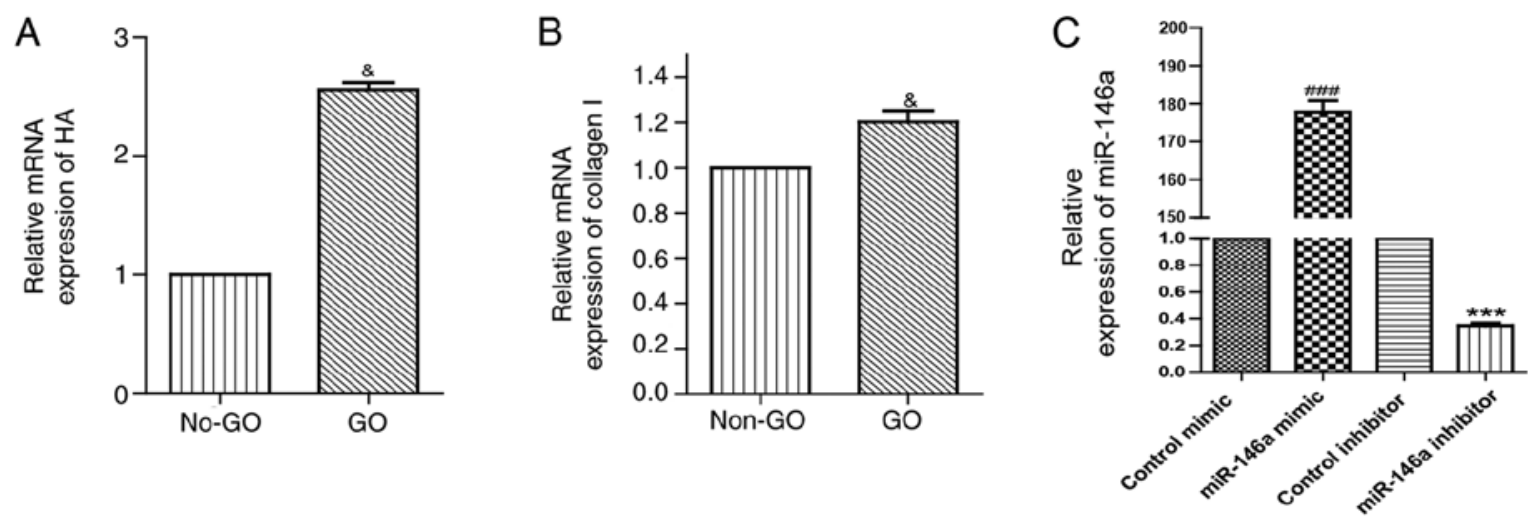

Figure 2. mRNA expression of HA and collagen I in orbital fibroblasts of GO and non-GO patients. mRNA expression of (A) HA and (B) collagen I in orbital fibroblasts from patients with GO and non-GO subjects. (C) Expression of miR-146a in orbital fibroblasts following infection with miR-146a mimics or inhibitor. Data are presented as the mean \pm standard deviation. ${ }^{\circledR} \mathrm{P}<0.05$ vs. non-GO; ${ }^{\# \#} \mathrm{P}<0.001$ vs. control mimic; ${ }^{* * *} \mathrm{P}<0.001$ vs. control inhibitor. HA, hyaluronic acid; GO, Graves' ophthalmopathy; miR, microRNA.

\section{Results}

Identification of fibroblasts. As presented in Fig. 1, vimentin exhibited a positive expression in the cytoplasm of orbital fibroblasts, indicating the mesenchymal origin of these cells. By contrast, desmin, myoglobin, keratin 17 and S100B were not expressed in these cells, excluding the possibility that these cells were smooth muscle cells, striated muscle cells, skin cells, nerve cells or skin melanocytes, which additionally indicated that the extracted cell population was not contaminated with other cell types. These results verified the identity of these cells as fibroblasts.

Expression of HA and collagen I in orbital fibroblasts. The mRNA expression level of HA in orbital fibroblasts of the GO group was approximately 2.6 folds higher compared with the non-GO group (Fig. 2A). Similarly, orbital fibroblasts from the GO group exhibited a higher expression level of collagen Ia2 mRNA compared with the non-GO group (Fig. 2B).
Infection efficiency of miR-146a mimics and inhibitor in orbital fibroblasts. RT-qPCR indicated that in the overexpression group, the expression level of miR-146a was $\sim 170$-folds higher compared with the control mimic, while miR-146a expression in the inhibition group was decreased to $\sim 30 \%$ of the control inhibitor expression level (Fig. 2C) suggesting that overexpression and inhibition of miR-146a in orbital fibroblasts were efficient. The morphology of orbital fibroblasts following infection was long fusiform or triangular, which was similar to the morphology of these cells before infection (Fig. 3). On the other hand, a green fluorescence signal was observed in $>80 \%$ (an approximation as observed under fluorescence microscopy) of fibroblasts post infection, which indicated a high level of infection efficiency (Fig. 3).

Effect of miR-146a on the expression of HA in GO orbital fibroblasts. As demonstrated in Fig. 4A, the mRNA level of HA in the miR-146a overexpression group was lower in comparison to control mimic, while the HA mRNA level in 

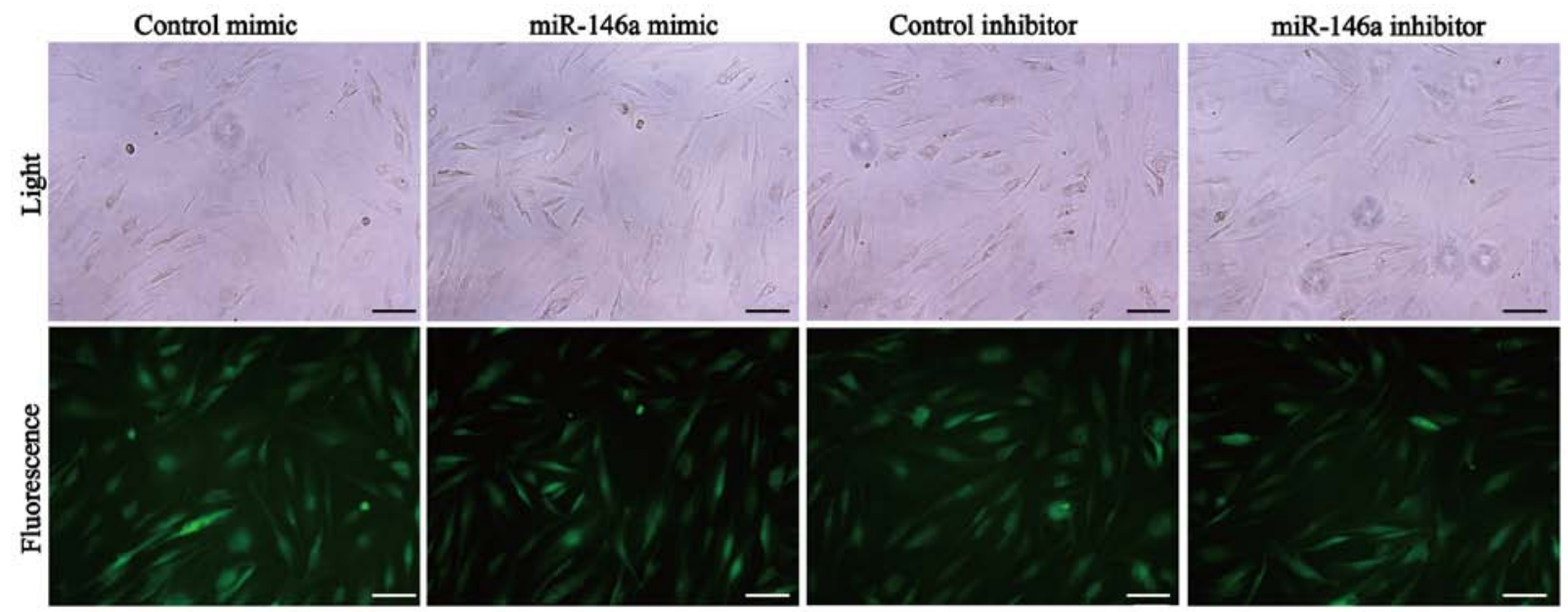

Figure 3. Morphology and infection efficiency of orbital fibroblasts. Under a light microscope, the morphology of fibroblasts infection with control or miR-146a mimic and control or miR-146a inhibitor was examined. The infection cells exhibited a green fluorescence under an inverted fluorescence contrast microscope at the same field of view. Scale bars, $100 \mu \mathrm{m}$. miR, microRNA.
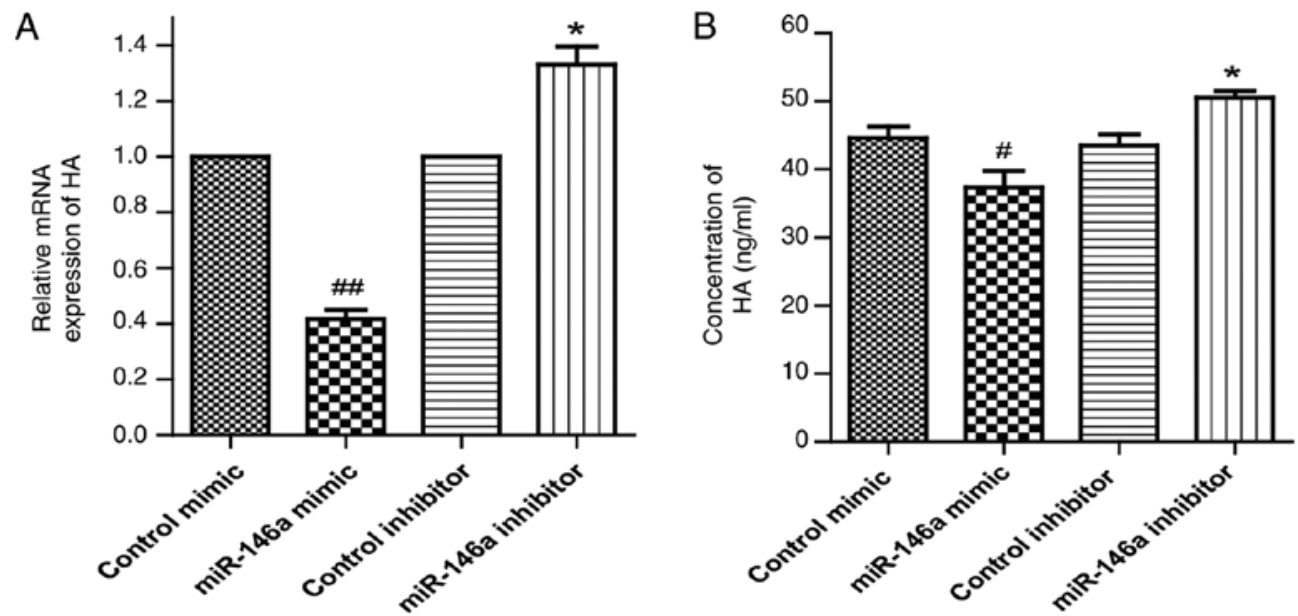

Figure 4. Effect of miR-146a on HA production in orbital fibroblasts. (A) mRNA and (B) protein levels of HA in orbital fibroblasts were detected via reverse transcription-quantitative PCR and ELISA, respectively. Data are presented as the mean \pm standard deviation $(\mathrm{n}=3) .{ }^{~} \mathrm{P}<0.05$ and ${ }^{\# \#} \mathrm{P}<0.01 \mathrm{vs}$. control mimic; ${ }^{*} \mathrm{P}<0.05$ vs. control inhibitor. HA, hyaluronic acid; miR, microRNA.

the miR-146a inhibitor group was higher compared with the control inhibitor. ELISA also indicated that overexpression of miR-146a inhibited HA secretion, while inhibition of miR-146a increased HA secretion in GO orbital fibroblasts compared with the respective control group (Fig. 4B).

Effect of miR-146a on the expression of collagen I in GO orbital fibroblasts. The mRNA level of collagen I in the miR-146a overexpression group was lower compared with control mimic, while the level of collagen I mRNA in the miR-146a inhibitor group was higher compared with control inhibitor (Fig. 5A). Consistently, ELISA results also indicated that overexpression of miR-146a inhibited, and inhibition of miR-146a increased the secretion of collagen I in GO orbital fibroblasts compared with the respective control group (Fig. 5B).

\section{Discussion}

In the current study, it was identified via IHC staining that the isolated primary cells were orbital fibroblasts, and a culture model of orbital fibroblasts was effectively generated. The results of the present study indicated that the mRNA levels of HA and collagen I in orbital fibroblasts from the GO cohort were higher compared with the non-GO cohort. Furthermore, miR-146a was efficiently overexpressed or inhibited in orbital fibroblasts. ELISA and RT-qPCR results revealed that the production of collagen I and HA was significantly reduced following overexpression of miR-146a, while inhibition of miR-146a significantly increased the production of collagen I and HA compared with the control group.

GO is a common eyelid disorder that seriously affects the appearance and visual function of patients, while severe GO may also result in blindness (23). GO has been indicated to be initiated with an autoimmune inflammatory response, HA synthesis and collagen deposition, resulting in connective tissue hyperplasia and fibrosis (24). Orbital fibroblasts have been revealed to be an important pathogenetic factor of GO, contributing to inflammation, lipogenesis, HA secretion and fibrosis in GO (24). HA is the principal component of GAG aggregation and exhibits high hydrophilicity binding to a large amount of water, thereby 

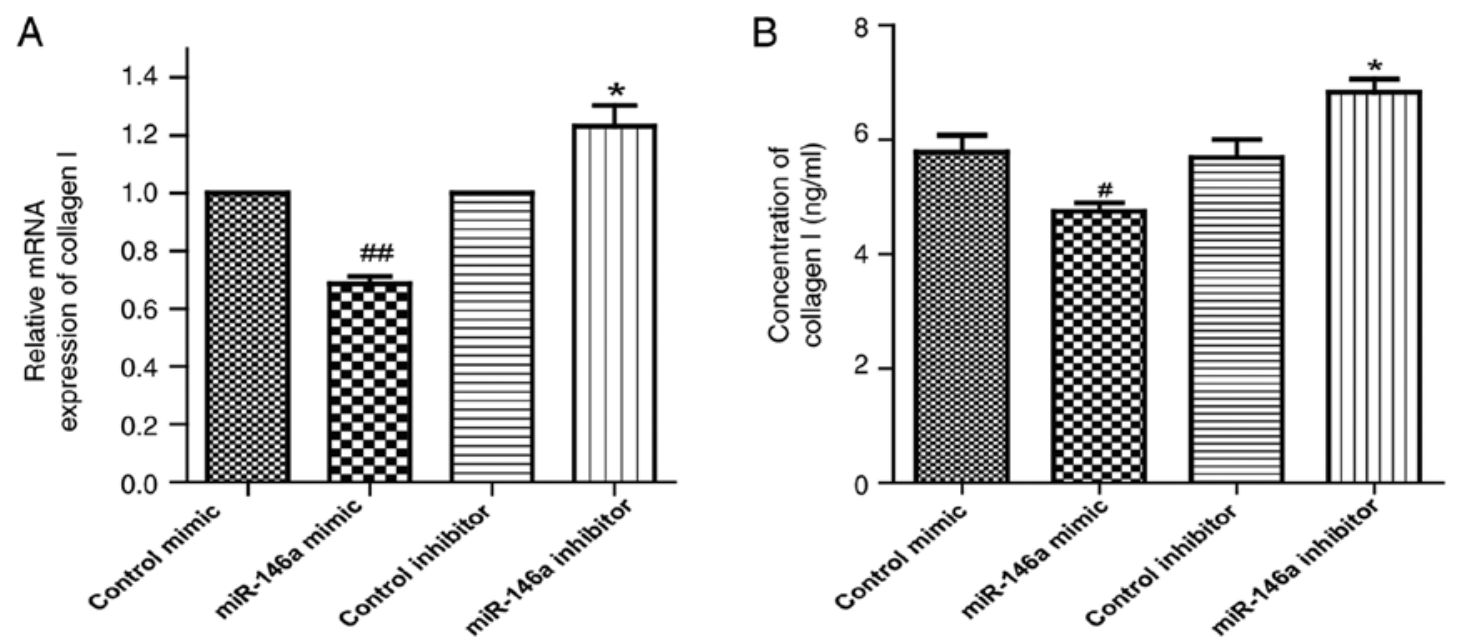

Figure 5. Effect of miR-146a on the secretion of collagen I in orbital fibroblasts. (A) mRNA and (B) protein levels of collagen I in orbital fibroblasts were detected via reverse transcription-quantitative PCR and ELISA, respectively. Data are presented as the mean \pm standard deviation ( $\mathrm{n}=3$ ). ${ }^{\prime \prime} \mathrm{P}<0.05$ and ${ }^{\# \#} \mathrm{P}<0.01$ vs. control mimic; ${ }^{*} \mathrm{P}<0.05$ vs. control inhibitor. miR, microRNA.

resulting in an abnormal eyelid tissue and extraocular muscle interstitial edema (25). The increase in HA production and collagen deposition has been indicated to increase the volume of orbital tissue in GO, thereby aggravating the symptoms of eyeball protrusion (26). The results of the current study are consistent with those of a previous study, which reported that miR-146a downregulated the expression of collagen I and was associated with TGF- $\beta$-mediated fibrosis (11). In addition, the effect of miR-146a on HA production was examined in the present study, and it was revealed that overexpression of miR-146a inhibited HA production, which suggests that upregulation of miR-146a may reduce GAG aggregation in patients with GO.

Numerous miRNAs have been indicated to serve regulatory roles in GO (27). miR-146a is a member of the miRNA family that regulates gene expression at the transcriptional level via downregulating its mRNA targets (13). Previous studies have indicated that miR-146a participated in several types of autoimmune diseases, including rheumatoid arthritis (17). In a previous report, miR-146a has been revealed to reduce the pathogenesis of GO via exerting anti-inflammatory and anti-fibrotic effects (11), while another study demonstrated that the upregulation of miR-146a promoted inflammation and disease progression (20). These studies have indicated that the effect of miR-146a on GO may be complex, as miR-146a may exert distinct functions in different cell types or interact with various target mRNAs. The present study only examined the role of miR-146a in primary cultures of GO orbital fibroblasts and additional in-depth investigations are required to verify the role and the molecular mechanisms of miR-146a function in animal models of GO.

In conclusion, the present study demonstrated that miR-146a downregulated the expression of HA and collagen I in GO orbital fibroblasts, which may reduce GAG aggregation and collagen deposition, thereby delaying disease progression. Therefore, miR-146a may represent a novel target for the treatment of GO. However, therapeutic applications based on miR-146a are not yet fully developed, and additional studies are required to examine the effects of miR-146a on other target genes or molecular mechanisms in GO.

\section{Acknowledgements}

Not applicable.

\section{Funding}

The present study was funded by National Natural Science Foundation of China (grant no. 81360152), Guangxi Natural Science Foundation (grant no. 2018GXNSFAA281234), 2019 Guangxi One Thousand Young and Middle-Aged College and University Backbone Teachers Cultivation Program and 'Medical Excellence Award' Funded by the Creative Research Development Grant from the First Affiliated Hospital of Guangxi Medical University.

\section{Availability of data and materials}

The datasets used and/or analyzed during the current study are available from the corresponding author on reasonable request.

\section{Authors' contributions}

KL proposed the current study and drafted the manuscript. WL designed and performed the experiments and wrote the manuscript. CM, HL, LC and SY collected and analyzed experimental data. All authors read and approved the final manuscript for publication.

\section{Ethics approval and consent to participate}

The current study was approved [approval no. 2019(KY-E-092)] by the Ethics Review Committee of The First Affiliated Hospital of Guangxi Medical University and written informed consent was obtained from all participants prior to inclusion.

\section{Patient consent for publication}

Not applicable. 


\section{Competing interests}

The authors declare that they have no competing interests.

\section{References}

1. Hiromatsu Y, Eguchi H, Tani J, Kasaoka M and Teshima Y: Graves' ophthalmopathy: Epidemiology and natural history. Intern Med 53: 353-360, 2014.

2. Wang L and Ma JM: Progression of the pathogenesis of thyroid associated ophthalmopathy. Zhonghua Yan Ke Za Zhi 53: 474-480, 2017 (In Chinese).

3. Gu LQ, Jia HY, Zhao YJ, Liu N, Wang S, Cui B and Ning G: Association studies of interleukin-8 gene in Graves' disease and Graves' ophthalmopathy. Endocrine 36: 452-456, 2009.

4. Park M, Banga JP, Kim GJ, Kim M and Lew H: Human placenta-derived mesenchymal stem cells ameliorate orbital adipogenesis in female mice models of Graves' ophthalmopathy. Stem Cell Res Ther 10: 246, 2019.

5. Bahn RS and Heufelder AE: Retroocular fibroblasts: Important effector cells in Graves' ophthalmopathy. Thyroid 2: 89-94, 1992.

6. Weetman AP, Cohen S, Gatter KC, Fells P and Shine B Immunohistochemical analysis of the retrobulbar tissues in Graves' ophthalmopathy. Clin Exp Immunol 75: 222-227, 1989.

7. Khong JJ, McNab AA, Ebeling PR, Craig JE and Selva D Pathogenesis of thyroid eye disease: Review and update on molecular mechanisms. Br J Ophthalmol 100: 142-150, 2016.

8. Kumar S, Coenen M, Iyer S and Bahn RS: Forkhead transcription factor FOXO1 is regulated by both a stimulatory thyrotropin receptor antibody and insulin-like growth factor-1 in orbital fibroblasts from patients with Graves' ophthalmopathy. Thyroid 25: 1145-1150, 2015.

9. Underhill CB: The interaction of hyaluronate with the cell surface: The hyaluronate receptor and the core protein. Ciba Found Symp 143: 87-99; discussion 100-106, 281-285, 1989.

10. van Steensel L, Paridaens D, Schrijver B, Dingjan GM, van Daele PL, van Hagen PM, van den Bosch WA, Drexhage HA, Hooijkaas H and Dik WA: Imatinib mesylate and AMN107 inhibit PDGF-signaling in orbital fibroblasts: A potential treatment for Graves' ophthalmopathy. Invest Ophthalmol Vis Sci 50: 3091-3098, 2009.

11. Jang SY, Park SJ, Chae MK, Lee JH, Lee EJ and Yoon JS: Role of microRNA-146a in regulation of fibrosis in orbital fibroblasts from patients with Graves' orbitopathy. Br J Ophthalmol 102: 407-414, 2018.

12. Wang Y and Smith TJ: Current concepts in the molecular pathogenesis of thyroid-associated ophthalmopathy. Invest Ophthalmol Vis Sci 55: 1735-1748, 2014.

13. Li K, Du Y, Jiang BL and He JF: Increased microRNA-155 and decreased microRNA-146a may promote ocular inflammation and proliferation in Graves' ophthalmopathy. Med Sci Monit 20: 639-643, 2014
14. Bhaskaran M and Mohan M: MicroRNAs: History, biogenesis, and their evolving role in animal development and disease. Vet Pathol 51: 759-774, 2014.

15. Bernardo BC, Ooi JYY, Lin RCY and McMullen JR: miRNA therapeutics: A new class of drugs with potential therapeutic applications in the heart. Future Med Chem 7: 1771-1792, 2015.

16. Wu F, Zikusoka M, Trindade A, Dassopoulos T, Harris ML, Bayless TM, Brant SR, Chakravarti S and Kwon JH: MicroRNAs are differentially expressed in ulcerative colitis and alter expression of macrophage inflammatory peptide- $2 \alpha$. Gastroenterology 135 : 1624-1635.e24, 2008.

17. Nakasa T, Miyaki S, Okubo A, Hashimoto M, Nishida K, Ochi M and Asahara H: Expression of microRNA-146 in rheumatoid arthritis synovial tissue. Arthritis Rheum 58: 1284-1292, 2008.

18. Tang Y, Luo X, Cui H, Ni X, Yuan M, Guo Y, Huang X, Zhou H, de Vries N, Tak PP, et al: MicroRNA-146A contributes to abnormal activation of the type I interferon pathway in human lupus by targeting the key signaling proteins. Arthritis Rheum 60: 1065-1075, 2009

19. Jang SY, Chae MK, Lee JH, Lee EJ and Yoon JS: Role of miR-146a in the regulation of inflammation in an in vitro model of Graves' orbitopathy. Invest Ophthalmol Vis Sci 57: 4027-4034, 2016.

20. Wang N, Chen FE and Long ZW: Mechanism of microrna-146a/ notch2 signaling regulating il-6 in Graves ophthalmopathy. Cell Physiol Biochem 41: 1285-1297, 2017.

21. Li H, Ma C, Liu W, He J and Li K: Gypenosides protect orbital fibroblasts in Graves ophthalmopathy via anti-inflammation and anti-fibrosis effects. Invest Ophthalmol Vis Sci 61: 64, 2020.

22. Livak KJ and Schmittgen TD: Analysis of relative gene expression data using real-time quantitative PCR and the 2(-Delta Delta $\mathrm{C}(\mathrm{T})$ ) method. Methods 25: 402-408, 2001.

23. Blandford Z, Zhang D, Chundury RV and Perry JD: Dysthyroid optic neuropathy: Update on pathogenesis, diagnosis, and management. Expert Rev Ophthalmol 12: 111-121, 2017.

24. Yang IH, Rose GE, Ezra DG and Bailly M: Macrophages promote a profibrotic phenotype in orbital fibroblasts through increased hyaluronic acid production and cell contractility. Sci Rep 9: 9622, 2019.

25. Iyer $S$ and Bahn R: Immunopathogenesis of Graves' ophthalmopathy: The role of the TSH receptor. Best Pract Res Clin Endocrinol Metab 26: 281-289, 2012.

26. Longo CM and Higgins PJ: Molecular biomarkers of Graves' ophthalmopathy. Exp Mol Pathol 106: 1-6, 2019.

27. Jang SY, Chae MK, Lee JH, Lee EJ and Yoon JS: MicroRNA-27 inhibits adipogenic differentiation in orbital fibroblasts from patients with Graves' orbitopathy. PLoS One 14: e0221077, 2019.

(i) $\Theta$ This work is licensed under a Creative Commons Attribution-NonCommercial-NoDerivatives 4.0 International (CC BY-NC-ND 4.0) License. 